\title{
A CASE OF ACUTE PSYCHOSIS FOLLOWING NORMALISATION OF THYROID STATUS WITH PROPYLTHIOURACIL IN LONG-STANDING HYPERTHYROIDISM
}

\author{
Tom Ansumol $^{1^{*}}$, Chacko Deenu ${ }^{2}$, Karunakaran Vidhukumar $^{3}$ \\ ${ }^{1}$ Junior Resident, ${ }^{2}$ Assistant Professor, ${ }^{3}$ Associate Professor, \\ Dept. of Psychiatry, Government Medical College, Ernakulam. \\ ${ }^{*}$ Correspondence: Department of psychiatry, Government Medical College, Ernakulam. E-mail:ansumoltom07@gmail.com
}

\begin{abstract}
Precipitation of acute psychosis following normalisation of thyroid status has been reported previously. Here, we report the case of a 62-year-old man with long-standing hyperthyroidism unresponsive to carbimazole, developing acute polymorphic psychotic disorder without symptoms of schizophrenia, along with normalization of thyroid status, after seven months of treatment with propylthiouracil.
\end{abstract}

Keywords: hyperthyroidism, acute psychosis, propylthiouracil

\section{INTRODUCTION}

The association between thyroid abnormalities and psychiatric disturbances is well known. Frank psychosis occurs in 10-20\% of hyperthyroid patients and in about $5 \%$ of hypothyroid patients. ${ }^{1}$ Abrupt normalisation of thyroid status, either from the hyperthyroid or the hypothyroid state, has been associated with precipitation of acute psychosis and mood disorders. ${ }^{2}$ The neurobehavioural effects of thyroxine may be related to its action on neurotransmitters. ${ }^{3}$ It has been shown that in rats with experimentally induced hypothyroidism, there is an increase in cerebral dopamine level and tyrosine hydroxylase activity. ${ }^{3}$ Here, we present the case of a 62-year-old man who while on treatment for long-standing hyperthyroidism presented with features of acute polymorphic psychotic disorder, and on investigation his thyroid status was found to have become normal for the first time.

\section{CASE SCENARIO}

A 62-year-old married male, without past or family history of any psychiatric illness, presented with acute onset of hearing voices and suspiciousness. He was hearing voices of people talking over the phone and believed that those voices were coming from a camera fitted in the room. He was suspicious that some illegal activities were going on there. He tried to inform the police, and he asked the nurses and hospital security staff to report the issues to the authorities. He later started to believe that the persons were arrested by the police as he complained about the issue. His mental status examination revealed increased amount of speech with circumstantiality, euphoric mood, delusion of persecution, and auditory hallucinations.

The patient had hyperthyroidism for past 20 years. Although he was on irregular treatment during initial years, there was regular follow-up and 
treatment with carbimazole for the past five years. Ultrasonography (USG) revealed that both lobes of thyroid were enlarged with coarse echotexture, and it was suggestive of thyroiditis. Fine Needle Aspiration Cytology (FNAC) suggested cellular follicular lesion and Hurthle cell change with mild focal atypia. His hyperthyroid status was persisting throughout these years, and finally, about seven months before the onset of psychosis, he was started on propylthiouracil $100 \mathrm{mg} \mathrm{BD}$. But his thyroid status did not become normal during the initial six months of treatment with propylthiouracil. But, when he was admitted in psychiatry ward following the onset of psychosis, his thyroid status was found to be normal for the first time.

There was no history of any substance use for past three years. He was on treatment for coronary artery disease and diabetes mellitus for past ten years and hypertension for past six years and was on regular medications. Routine blood investigations, serum electrolytes, and CT brain were normal.

The patient was treated with $\mathrm{T}$. risperidone $1 \mathrm{mg}$ BD. His symptoms subsided within three days and the antithyroid medications were continued. After discharge, the patient was on regular follow-up for three months. He was having no features of psychosis while on follow-up and subsequently he was lost from follow-up.

\section{DISCUSSION}

Normalization of thyroid status resulting in precipitation of acute psychosis was reported previously also. Our patient had long-standing and severe hyperthyroidism which was not corrected with carbimazole, and so propylthiouracil was added. After adding propylthiouracil, his thyroid status became normal for the first time. Simultaneously, he developed psychosis which was acute in onset. Thus, it is possible that the normalisation of thyroid status by treatment with propylthiouracil precipitated the psychosis.

Thyroid hormones are widely distributed in the brain. ${ }^{4}$ They are crucial during the development of the fetal brain and also for maintenance of adult brain function. ${ }^{4}$ They are essential for normal differentiation of cells and metabolic balance. ${ }^{5}$ Along with different effects on the central nervous system, they also have a role in the pathogenesis of psychiatric disorders. ${ }^{4}$ Abnormalities in the thyroid hormone levels can have an impact on the manifestation of psychiatric disorders. ${ }^{6}$ It has been shown that thyroid hormones can regulate the level of dopamine receptors in the brain and also the activity of tyrosine hydroxylase, the rate-limiting enzyme of the catecholamine pathway which is involved in the synthesis of dopamine also. ${ }^{4}$ Dopamine is the major neurotransmitter implicated in the pathogenesis of psychosis. ${ }^{4}$ Thyroid hormones also enhance or suppress the transcription of numerous genes. ${ }^{7}$ There is a possibility that a rapid reduction in high ambient thyroid hormone which occurs following rapid normalization of thyroid hormone levels could lead to alteration or recovery of cellular function at different rates in different regions of the brain. ${ }^{7}$ These factors might have triggered the acute psychosis in this patient. New-onset psychosis has been reported in a patient who underwent radioactive iodine treatment for thyroid carcinoma which resulted in acute hypothyroidism. ${ }^{8}$

\section{CONCLUSION}

Normalisation of thyroid status from long-standing hyperthyroid status can result in precipitation of acute psychosis. So, the treating physician should be vigilant while rapidly normalising thyroid levels.

\section{REFERENCES}

1. Benvenga S, Lapa D, Trimarchi F. Dont forget the thyroid in the etiology of psychoses. Am J Med 2017; 115(2):159-60.

2. Gowda G, Sagi M, Komal S, Jaisoorya T. Psychosis following carbimazole-induced acute alteration of hyperthyroid status. Indian J Psychol Med 2017; 39(4):516. Sharma P, Parmar C, Parikh N. A case report on myxedema madness: Curable psychosis. Indian J Psychol Med 2014; 36(1):80-1.

3. Santos NC, Costa P, Ruano D, Macedo A, Soares MJ, Valente J, et al. Revisiting thyroid hormones in schizophrenia. J Thyroid Res 2012; 2012:569147.10.1155/2012/569147 
4. Zhang J, Lazar MA. The mechanism of action of thyroid hormones. Annu Rev Physiol 2000; 62(1):439-66.

5. Radhakrishnan R, Calvin S, Singh JK, Thomas B, Srinivasan K. Thyroid dysfunction in major psychiatric disorders in a hospital based sample. Indian J Med Res 2013; 138(6):888-93.

6. Irwin R, Ellis PM, Delahunt J. Psychosis following acute alteration of thyroid status. Aust $\mathrm{N} \mathrm{Z} \mathrm{J}$ Psychiatry1997; 31(5):762-4.

7. Freeman SA. Radioactive Iodine-induced psychosis. Am J Psychiatry 2009; 166(9):1067-8.

Source of support: None

Conflict of interest: None declared

First submitted: $15^{\text {th }}$ December 2017

Published online: $6^{\text {th }}$ March 2018 